\title{
Ruprecht 3: An old star cluster remnant?`
}

\author{
D. B. Pavani ${ }^{1}$, E. Bica ${ }^{1, \star \star}$, A. V. Ahumada ${ }^{2}$, and J. J. Clariá ${ }^{2}$ \\ 1 Universidade Federal do Rio Grande do Sul, IF, CP 15051, Porto Alegre 01501-970, RS, Brasil \\ 2 Observatorio Astronómico de Córdoba, Laprida, 854, 5000, Córdoba, Argentina \\ e-mail: andrea@mail.oac.uncor.edu; claria@mail.oac.uncor.edu
}

Received 14 October 2002 / Accepted 20 November 2002

\begin{abstract}
MASS $J$ and $H$ photometry and integrated spectroscopy are employed to study the nature of the poorly populated compact concentration of stars Ruprecht 3 , which was previously catalogued as an open cluster. The integrated spectrum remarkably resembles that of a moderately metal-rich globular cluster. The distribution of the object stars in the colour-magnitude diagram is compatible with that of a $1.5 \pm 0.5 \mathrm{Gyr}$ open cluster or older, depending on whether the bluer stars are interpreted as turnoff stars or blue stragglers, respectively. We derive for the object a distance from the Sun $d_{\odot}=0.72_{-0.03}^{+0.04} \mathrm{kpc}$ and a colour excess $E(B-V)=0.04$. Although a globular cluster remnant cannot be ruled out, the integrated spectrum resemblance to that of a globular cluster probably reflects a stochastic effect owing to the few brighter stars. The structural and photometric properties of Ruprecht 3 are compatible with what would be expected for an intermediate-age open cluster remnant.
\end{abstract}

Key words. Galaxy: open clusters and stellar associations: general - open clusters and associations: individual: Ruprecht 3 methods: observational - techniques: spectroscopic

\section{Introduction}

There has been recently growing interest in investigating open star clusters and their remnants. In the wake of this tendency, studies based on numerical simulations (de la Fuente Marcos 1997, 1998) have shown that the open cluster lifetime and stellar content depend on the initial mass function, fraction of primordial binaries and galactocencentric distance. The final cluster remnant is expected to be rich in binary stars. Additionally, remnants of poorly populated clusters are expected to be young, containing early-type stars, while remnants of rich open clusters are expected to contain later-type stars. It is also within reasonable expectations to assume that owing to the effect of mass segregation, open cluster remnants should present depletion of low Main Sequence (MS) stars by means of encounters with more massive stars and binary ones and evaporation due to the tidal field of the Galaxy. The depletion of low MS stars would imply that clusters which are close to disruption, have a core rich in compact and giant stars (Takahashi $\&$ Portegies Zwart 2000). From the observational point of view, an open cluster remnant can be defined as a poorly populated

Send offprint requests to: D. B. Pavani,

e-mail: dpavani@if.ufrgs.br

* Based on observations made at Complejo Astronómico El Leoncito, which is operated under agreement between the Consejo Nacional de Investigaciones Científicas y Técnicas de la República Argentina and the National Universities of La Plata, Córdoba and San Juan, Argentina.

$\star \star$ e-mail: bica@if.ufrgs.br concentration of stars resulting from the dynamical evolution of an initially more massive system (Pavani et al. 2001). There is currently existing knowledge about the final destiny of open clusters. The nature and dynamical stage of some stellar groups have been inferred. Despite these facts, however, only a few of them have been studied in detail and open questions still remain. For instance, NGC 6994 (M73) has been either interpreted as a possible remnant of an old open cluster or, alternatively, as a random fluctuaction of the star density in the object region (Bassino et al. 2000; Carraro 2000; Ordenkirchen \& Soubiran 2002). Recently, NGC 7036 and NGC 7772 were analysed by Carraro (2002), whose study suggests that both objects are open cluster remnants (OCRs). NGC 7036 is a stellar group with 17 likely members located at $1 \mathrm{kpc}$ from the Sun. It has a core radius of about 3-4 arcmin and an age of 3-4 Gyr. NGC 7772, located at about $1.5 \mathrm{kpc}$ from the Sun, was found to have 14 likely members with an age of $1.5 \mathrm{Gyr}$. Many other candidates exist, e.g. in the study of Bica et al. (2001), where an analysis of number density contrast has been performed. In a study of the stellar group NGC 1252, Pavani et al. (2001) favoured an open cluster remnant. Likely members populate evolutionary sequences in the colour-magnitude diagram (CMD). In the present study we discuss the case of the stellar group Ruprecht 3 .

Ruprecht 3, also known as OCL-642 (Alter et al. 1970) or ESO 426SC33 (Lauberts 1982), is located in Canis Major at $\mathrm{J} 2000.0 \alpha=06^{\mathrm{h}} 42^{\mathrm{m}} 07^{\mathrm{s}}, \delta=-29^{\circ} 27^{\prime} 15^{\prime \prime}\left(l=238^{\circ} .77, b=\right.$ $-14^{\circ} .81$ ). This object was classified as II1p in the Trumpler System by Ruprecht (1966), i.e. as an open cluster with 
the second highest concentration degree, a small range of brightness of its stars and a scanty population. The direction towards Ruprecht 3 was probed in H I by Olano et al. (1980) but none was found to be related to the object. We brought Ruprecht 3 under the focus of our investigation on account of its status as a relatively compact concentration of stars of $\approx 3^{\prime}$, similar to those analysed in Bica et al. (2001). The object region is already available in the Two Micron All Sky Survey (2MASS) photometric catalogue http://www . ipac. caltech. edu/2mass/. In Sects. 2 and 3 we present the photometry and discuss structural properties of Ruprecht 3, respectively. In Sect. 4 we describe the spectroscopic observations and reductions. An interpretation of the turnoff stars is discussed in Sect. 5, while the concluding remarks of the present study are given in Sect. 6 .

\section{Photometry}

In Fig. 1 we present a second generation Digitized Sky Survey (XDSS) $R$ image of Ruprecht 3 . The object stands out significantly from the background. It includes 23 stars which will be used in the subsequent analysis. Out of them 14 stars possess photometry in the $J, H$ bands in the 2MASS catalogue. This survey employed two 1.3-m telescopes, one at Mt. Hopkins (Arizona) and another at Cerro Tololo InterAmerican Observatory (CTIO, Chile). The telescopes were equipped with a three-channel camera, each channel consisting of a $256 \times 256$ array of $\mathrm{HgCdTe}$ detectors, capable of observing the sky simultaneously at $J(1.25 \mu \mathrm{m}), H(1.65 \mu \mathrm{m})$, and $K_{s}(2.17 \mu \mathrm{m})$. We made use of 2MASS data from the Second Incremental Release. We employed the Point Source Catalogue (PSC) through the GATOR access. Positions, magnitudes and photometric errors were provided. The adopted total errors are root-sum-square combinations of the photometric errors, the nightly zero-point, the estimated flat-fielding residual (0.005 mag), and the photometric normalization uncertainty for bright sources. In Fig. 2 we show the total photometric errors for the $H$ magnitude $\left(\sigma_{H}\right)$ and $(J-H)$ colour $\left(\sigma_{(J-H)}\right)$ as a function of $H$ magnitude, where $\sigma_{(J-H)}$ is $\sqrt{\sigma_{J}^{2}+\sigma_{H}^{2}}$. We can see that the errors attain $0.1 \mathrm{mag}$ at $H \approx 15.0$.

\section{1. $H \times(J-H) C M D$}

In Fig. 3 (left panel) we present the $H \times(J-H)$ CMD for Ruprecht 3, containing 14 stars and the respective total colour error bars (Sect. 2). The extraction corresponds to a diameter of $4^{\prime}$ centered on the object. In this diagram and subsequent ones, we eliminated non-stellar sources as indicated in the Guide Star Catalogue (GSC2.2) (http://vizier.u-strasbg.fr/vizbin/VizieR?-source $=\mathrm{I} / 271$ ). We employed solar metallicity Padova isochrones (Girardi et al. 2000) of different ages together with reddening constraints to find possible solutions. We used the relation $A_{H}=0.177 A_{V}$ (Schlegel et al. 1998), $R_{V}=3.1$ (Cardelli 1989) and the spectroscopic value $E(B-V)=0.04$ (Sect. 4) to obtain $A_{H}=0.02$. We derived from data in Schlegel et al. (1998) the relation $E(J-H)=0.33 E(B-V)$, which implies $E(J-H)=$ 0.013 in our case. From the isochrone fit the resulting

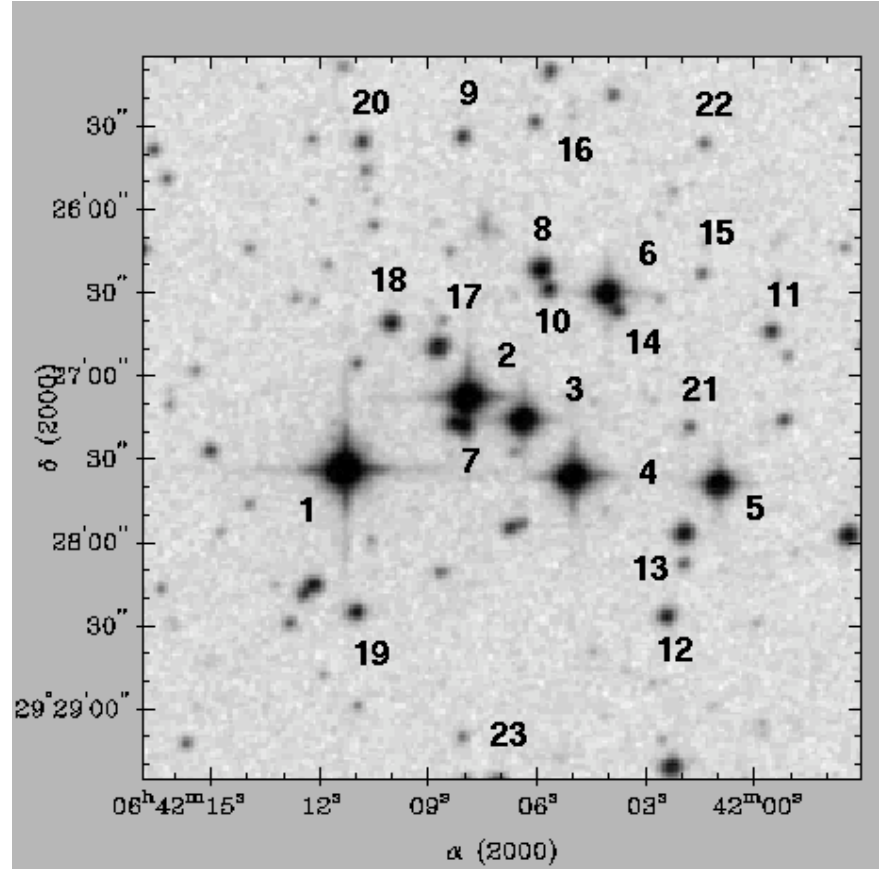

Fig. 1. 4.3 $\times 4.3 \operatorname{arcmin}^{2}$ XDSS $R$ image of Ruprecht 3. Labels identify studied stars.

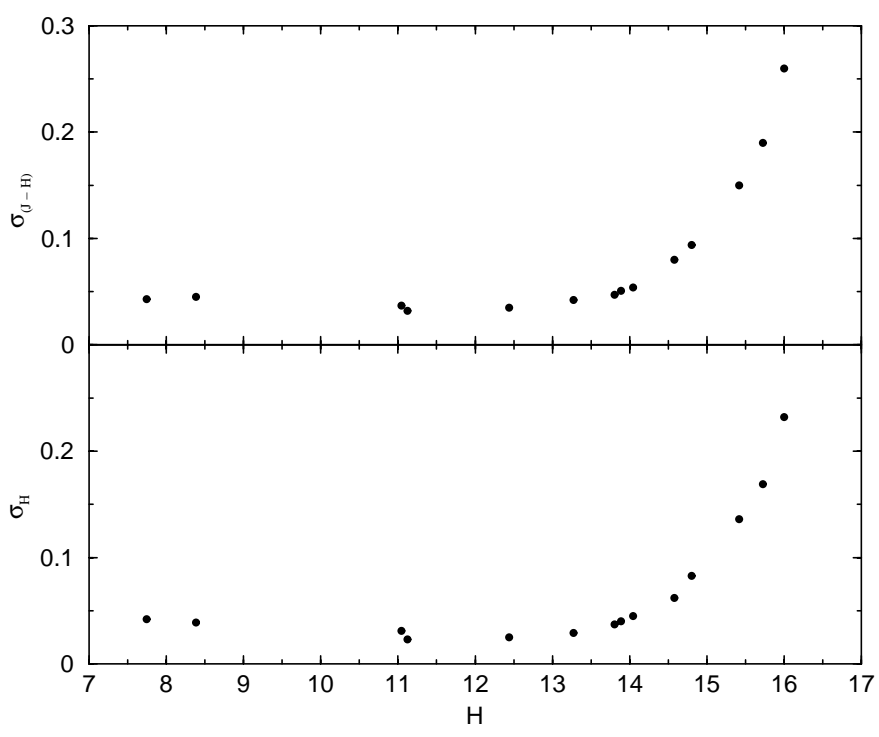

Fig. 2. $(J-H)$ total photometric errors (upper panel) and those in $H$ (lower panel) as a function of $H$ magnitude.

absolute distance modulus is $(m-M)_{o}=9.38$. Isochrones in the range 1-3 Gyr are consistent with the loci of bright stars and an approximate envelope to the fainter stars.

In the central panel of Fig. 3 we show an offset field $\left(\approx 12^{\prime}\right.$ north) with a diameter equal to that of the object extraction field. The stars in the offset field are distributed in a bluer and fainter region with a counterpart in the left panel, thus offering the possibility of discriminating members and field stars in the direction of Ruprecht 3.

In order to remove the contamination of field stars in the Ruprecht 3 CMD, we employed a statistical method which consists in comparing the distribution of stars in the object CMD with that of the representative field stars. Such comparison is 
based on the hypothesis that the positions of the stars in the field CMD represent the most likely loci for probable field stars on the object CMD. In this way, we try to estimate the probability of each star in the object extraction being a field star, and according to this probability we randomly remove stars from the object CMD. This method is described in detail in Kerber et al. (2002). With the purpose of improving the comparison field statistics, we extracted stars in a large area corresponding to an annulus $8^{\prime}<r<10^{\prime}$ centred on the object. Prior to the statistical removal, the number of stars in the object and field areas were scaled.

In Fig. 3 (right panel) we show the results of the subtraction for four realizations. The faint blue stars are removed in any realization. For the remaining stars, different symbols suggest membership according to the number of realizations in which the star was not removed. Note that such stars provide a good fit to the Padova isochrones, suggesting that Ruprecht 3 is a physical system.

From the position of the stars in the left and right panels of Fig. 3, we inferred probable members and non-members as indicated in Table 1. By Cols.: (1) running number for stars in the area of Ruprecht 3, (2) and (3) designations in the GSC2.2 and 2MASS catalogues, respectively, (4) and (5) J2000.0 coordinates, (6) and (7) 2MASS photometry, (8) and (9) USNO photometry (Sect. 2.2), (10) spectral types (Sect. 4), and finally (11) membership status.

\section{2. $R \times(B-R)$ photographic $C M D$}

The bright stars 2, 3 and 7 (Fig. 1) are unfortunately absent in the 2MASS photometry (Table 1). The photographic photometry in the $B$ and $R$ bands is available in the USNO A2.0 catalogue (http://vizier. u-strasbg.fr/viz-bin/VizieR?-source=I/252) for most stars in the area of Ruprecht 3 (Table 1). This provides us with a means to check membership status for star 3 and some additional faint ones. We have also checked the photographic photometry from GSC2.2, where most bright stars are absent in $R$, but the intermediate and faint stars are complete. The stars in common between USNO and GSC2.2 show a good photometric agreement, and star 7 (Table 1), which is absent in USNO, had its value adopted from GSC2.2. In Fig. 4 we show the resulting $R \times(B-R) \mathrm{CMD}$. The Padova isochrone fit is compatible with that in the infrared (left panel of Fig. 3). We used $A_{R}=0.82 A_{V}$ (Schlegel et al. 1998) thus obtaining $A_{R}=0.10$. The relation $E(B-R)=1.563 E(B-V)$ was also obtained, and a reddening value $E(B-R)=0.06$ was derived. The resulting absolute distance modulus turns out to be $(m-M)_{o}=9.4$. Note that the faint blue stars appear to be field contamination stars as in Fig. 3. The distribution of the bright stars is compatible with that of a giant branch. Using this information, we inferred membership status for some bright and faint stars which were absent in the 2MASS photometry (Table 1).

\section{Structural properties}

In Fig. 5 we present the stellar surface density profiles and corresponding Poisson error bars as derived from the 2MASS and USNO catalogues. We obtained the surface densities by performing star counts in concentric annuli centred at the object and then dividing by their respective surface areas. For each photometric system, we took into account all measured stars. The surface density profiles have a peak for the central arcmin, after which the profiles appear to be flat. We performed a background analysis in offset fields $15^{\prime}$ north for 2MASS and $15^{\prime}$ south-east for USNO data. The fields were circular with $r=4^{\prime}$ and the derived stellar surface density values are indicated as dashed lines in Fig. 5.

Since the 2MASS photometry lacks a few bright stars the peak is less pronounced. The presence of some additional stars in the USNO profile makes the peak statiscally more significant. Both panels of Fig. 5 show that the object is compact with a maximum core radius of $1^{\prime}$. The values for the annuli outside the central region are comparable to those of the offset fields and, consequently, the object does not appear to have extensions, which is not unlikely in the case of an open cluster remnant.

\section{Spectroscopy}

\subsection{Observations}

The spectroscopic observations of Ruprecht 3 were carried out with the 2.15-m telescope at the Complejo Astronómico El Leoncito (CASLEO) in San Juan (Argentina) during two observing runs in November and December 2001, respectively. In both runs we employed a $C C D$ camera containing a Tektronics chip of $1024 \times 1024$ pixels attached to the REOSC spectrograph (simple mode), the size of each pixel being $24 \mu \mathrm{m} \times$ $24 \mu \mathrm{m}$; one pixel corresponds to $0.94^{\prime \prime}$ on the sky. The long slit, corresponding to $4.7^{\prime}$ on the sky, allowed us to sample regions of the background sky. The seeing during the nights was typically $2.0^{\prime \prime}$. A grating of 300 grooves $/ \mathrm{mm}$ was used. The spectral coverage was (3600-6900) $\AA$, with an average dispersion in the observed region of $\approx 138 \AA / \mathrm{mm}(3.3 \AA / \mathrm{pix})$. The slit width was $4.5^{\prime \prime}$, resulting in a resolution of $\approx 17 \AA$, as measured by the mean full width half maximum (FWHM) of the comparison lines. In total, we took 3 exposures of the object of 15 min each and 3 of 10 min with the aim of having an acceptable signal-to-noise ratio and correcting for cosmic rays. Three standard stars from the list of Stone \& Baldwin (1983) were observed for flux calibrations, and $\mathrm{He}-\mathrm{Ne}-\mathrm{Cu}$ lamp exposures were taken for wavelength calibrations.

The slit was set in the east-west direction and the observations were performed scanning the slit across the object in the north-south direction in order to sample the stellar content adequately. Then, the slit was set at position angle $45^{\circ}$ in order to allow spatial extractions corresponding to the bright stars1-4 (Fig. 1). In particular, spectroscopic information of star 2 is required owing to the lack of 2MASS and USNO photometry (Table 1).

The reductions were carried out at the Observatorio Astronómico de Córdoba (Argentina), using the IRAF package 


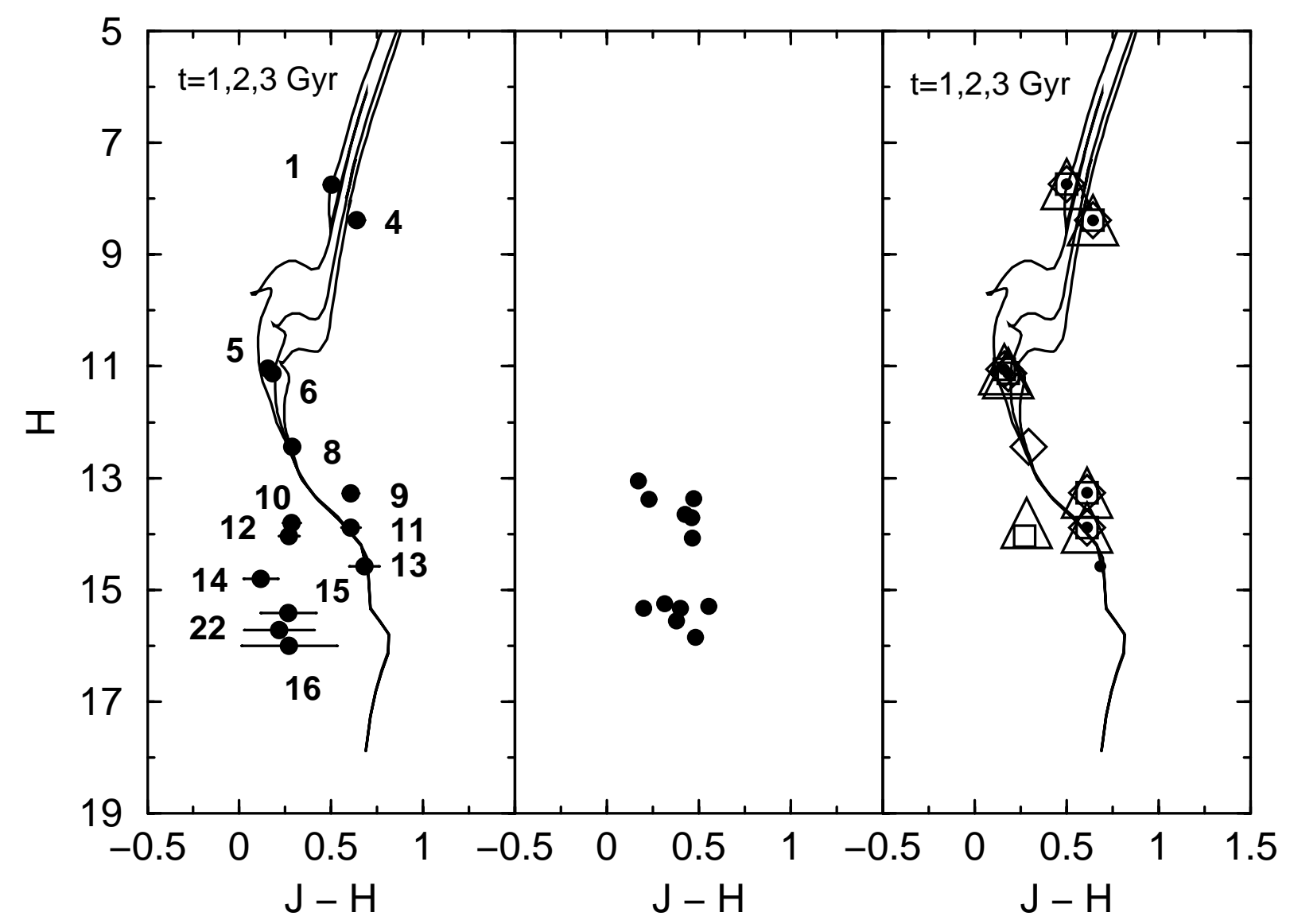

Fig. 3. Left panel: $H \times(J-H)$ diagram for 14 stars in the area of Ruprecht 3 with 2 MASS photometry. Middle panel: offset field (12' north) with same diameter as that of Ruprecht 3. Right panel: result of statistical subtraction: each symbol corresponds to member in a given realization. Padova isochrones are shown.

and following standard procedures. We subtracted the bias and used flat-field frames - previously combined - to correct the frames for high and low spatial frequency variations. Background sky subtractions were then performed using pixel rows from the same frame after removing cosmic rays from the background sky regions. We ensured that no significant background sky residuals were present on the resulting spectra. Wavelength calibrations were carried out with a $\mathrm{Cu}-\mathrm{He}-\mathrm{Ar}$ lamp with exposures following that of the object or standard star. Atmospheric extinction corrections according to the site coefficients given by Minniti et al. (1989) and flux calibrations were then applied.

\subsection{Individual stars}

The individual spectra of stars 1 to 4 are shown in Figs. 6 and 7. Although they all appear to be late-type stars, we employed the stellar templates library by Silva \& Cornell (1992) to assign spectral types with greater detail. In Fig. 7 we illustrate the comparison of the reddening-corrected spectrum of star 1 of Ruprecht 3 with the G8K0II template that best matches this spectrum. Although the present spectral resolution is too low for determining luminosity classes and it is possible to determine only spectral types, most best matches occurred for giant templates in the library of Silva \& Cornell (1992). The results obtained for stars $2-4$ are given in Table 1.
Note that their spectral types range from G2 to K5, which is expected for giants in Ruprecht 3 . We used these results as additional membership criteria, in particular for star 2 (Table 1). Simultaneously to the comparison with the template, we estimated the amount of interstellar reddening affecting each star. Thus we found $E(B-V)=0.02,0.05,0.03$ and 0.11 magnitudes for stars $1,2,3$ and 4 , respectively. The resulting average is $E(B-V)=0.05 \pm 0.04$.

\subsection{Integrated spectroscopy}

The integrated spectra of Ruprecht 3 obtained in the two different runs were averaged by weighting them according to the signal-to-noise ratio and the result is shown in Fig. 8 (bottom). The spectrum was compared with star cluster templates from Bica (1988). As shown in Fig. 8, the integrated spectral features of Ruprecht 3 appear to be remarkably similar to that of a relatively metal-rich globular cluster (template G2, $[\mathrm{Fe} / \mathrm{H}]=-0.5)$. The best template match is found correcting the observed spectrum of Ruprecht 3 by $E(B-V)=0.04$. We adopted this reddening value for the object, which is in very good agreement with that obtained from the individual giants (Sect. 4.2).

Although a globular cluster remnant cannot be ruled out, the integrated spectrum resemblance to that of a globular 
Table 1. Stars in the area of Ruprecht 3.

\begin{tabular}{|c|c|c|c|c|c|c|c|c|c|c|}
\hline Rup 3 & $\mathrm{GSC} 2.2^{\mathrm{a}}$ & $2 \mathrm{MASS}^{\mathrm{b}}$ & $\begin{array}{r}\alpha_{2000} \\
\mathrm{~h}: \mathrm{m}: \mathrm{s}\end{array}$ & $\begin{array}{l}\delta_{2000} \\
{ }^{\prime}::^{\prime \prime}\end{array}$ & $\begin{array}{r}H \\
2 \mathrm{MASS}\end{array}$ & $\begin{array}{r}J \\
2 \mathrm{MASS}\end{array}$ & $\begin{array}{r}B \\
\text { USNO }\end{array}$ & $\begin{array}{r}R \\
\text { USNO }\end{array}$ & ST & membership $^{c}$ \\
\hline 1 & S101331196 & 0642113-292732 & $06: 42: 11.3$ & $-29: 27: 32$ & 7.74 & 8.24 & 11.40 & 9.40 & G8K0 & $\mathrm{pm}$ \\
\hline 2 & S87101331187 & - & 06:42:07.9 & $-29: 27: 07$ & - & - & - & 10.08 & G8K0 & $\mathrm{pm}$ \\
\hline 3 & S101331191 & - & $06: 42: 06.4$ & $-29: 27: 16$ & - & - & 11.80 & 11.10 & G2 & $\mathrm{pm}$ \\
\hline 4 & S101331198 & 0642050-292736 & $06: 42: 05.0$ & $-29: 27: 36$ & 8.38 & 9.02 & 12.00 & 10.60 & K5 & $\mathrm{pm}$ \\
\hline 5 & S1013311101 & 0642010-292740 & $06: 42: 01.0$ & $-29: 27: 40$ & 11.05 & 11.20 & 12.00 & 11.90 & - & lpm \\
\hline 6 & S101331173 & 0642039-292630 & $06: 42: 04.0$ & $-29: 26: 31$ & 11.12 & - & - & - & - & $\mathrm{pm}$ \\
\hline 7 & S101331118740 & - & 06:42:08.1 & $-29: 27: 16$ & - & - & $14.02^{\mathrm{d}}$ & $13.47^{\mathrm{d}}$ & - & $\mathrm{pm}$ \\
\hline 8 & S101331170 & 0642058-292621 & $06: 42: 05.8$ & $-29: 26: 22$ & 12.44 & 12.72 & 13.50 & 12.60 & - & pm \\
\hline 9 & S101331119106 & 0642079-292533 & $06: 42: 07.9$ & $-29: 27: 34$ & 13.27 & 13.88 & 18.40 & 17.80 & - & $\mathrm{pm}$ \\
\hline 10 & S101331172 & 0642056-292629 & $06: 42: 05.6$ & $-29: 26: 29$ & 13.80 & 14.09 & - & - & - & lpm \\
\hline 11 & S101331118722 & 0641594-292645 & $06: 41: 59.5$ & $-29: 26: 46$ & 13.89 & 14.49 & 17.10 & 15.80 & - & $\mathrm{pm}$ \\
\hline 12 & S101331118137 & 0642025-292827 & $06: 42: 02.5$ & $-29: 28: 27$ & 14.04 & 14.31 & 16.10 & 15.20 & - & lpm \\
\hline 13 & S101331118310 & 0642020-292808 & $06: 42: 02.0$ & $-29: 28: 08$ & 14.58 & 15.26 & 18.40 & 17.00 & - & $\mathrm{pm}$ \\
\hline 14 & - & 0642037-292637 & $06: 42: 03.7$ & $-29: 26: 37$ & 14.81 & 14.92 & - & - & - & pnm \\
\hline 15 & S101331118851 & 0642013-292624 & $06: 42: 01.4$ & $-29: 26: 24$ & 15.42 & 15.69 & 18.30 & 17.10 & - & pnm \\
\hline 16 & S101331119131 & 0642059-292529 & $06: 42: 05.9$ & $-29: 25: 29$ & 15.72 & 15.94 & 17.40 & 16.70 & - & pnm \\
\hline 17 & S101331118741 & - & $06: 42: 08.7$ & $-29: 26: 49$ & - & - & 15.50 & 14.10 & - & $\mathrm{pm}$ \\
\hline 18 & S101331118759 & - & 06:42:09.9 & $-29: 26: 40$ & - & - & 16.00 & 15.10 & - & lpm \\
\hline 19 & S101331118144 & - & $06: 42: 11.1$ & $-29: 28: 24$ & - & - & 16.00 & 15.10 & - & lpm \\
\hline 20 & S101331119100 & - & $06: 42: 10.6$ & $-29: 25: 35$ & - & - & 16.80 & 16.10 & - & pnm \\
\hline 21 & S101331118512 & - & $06: 42: 01.8$ & $-29: 27: 20$ & 15.42 & 15.69 & 18.30 & 17.10 & - & pnm \\
\hline 22 & S101331119088 & 0642012-292537 & $06: 42: 01.3$ & $-29: 25: 38$ & 16.00 & 16.27 & 17.90 & 17.20 & - & pnm \\
\hline 23 & S101331117846 & - & 06:42:08.2 & $-29: 29: 09$ & - & - & 18.10 & 17.30 & - & pnm \\
\hline
\end{tabular}

${ }^{\mathrm{a}}$ GSC: Guide Star Catalogue; ${ }^{\mathrm{b}}$ MASS: Two Micron All Sky Survey; ${ }^{\mathrm{c}} \mathrm{mm}$, means probable, lpm, less probable and pnm, probable non-member;

${ }^{\mathrm{d}}$ Adopted from the GSC2.2 photometry.

cluster probably reflects a stochastic effect owing to the few brighter stars in the CMDs (Fig. 3, right panel, and Fig. 4).

\section{Discussion}

Proper motions from the Tycho-2 Catalogue (http:// vizier.hia.nrc.ca/viz-bin/VizieR?-source=I/259/ tyc2) are available for only five stars in the area of Ruprecht 3 (Fig. 1), but the results are worth discussing. We show in Fig. 9 $\mu_{\alpha} \times \mu_{\delta}$ and respective errors. Four stars (1-4) appear to share common motion, while star number 5 is offset. We cannot exclude it as a member owing to the possibility of binarity. Indeed, the Tycho- 2 observations span a few years. At any rate, based on this result, we classified star 5 as a less probable member (Table 1). Ideally, plates from early years in the XX century like $\mathrm{ACT}^{\prime}$ 's combined with recent images, can remove the uncertainty, except for binaries with periods longer than 100-200 yr (Baumgardt 1998).

In Fig. 10 we present the absolute CMD $M_{H} \times(J-H)_{o}$, where we superimposed the result of field subtraction for Ruprecht 3 (Fig. 3, right panel) on the distribution of stars for the old open cluster M67. The absolute CMD was built in two steps. We first extracted from 2MASS data which correspond to a diameter of $16^{\prime}$ centred on M $67\left(\alpha=08^{\mathrm{h}} 51^{\mathrm{m}} 21^{\mathrm{s}}\right.$, $\left.\delta=+11^{\circ} 49^{\prime} 50^{\prime \prime}\right)$. Subsequently, we adopted the reddening value $E(J-H)=0.00$ and the absolute distance modulus
$(m-M)_{0}=9.3$ (Pavani 2001). The same procedure was applied to Ruprecht 3 adopting $A_{H}=0.02$ and $E(J-H)=0.013$. We then matched the CMD of Ruprecht 3 to that of M 67, thereby deriving an absolute distance modulus $(m-M)_{0}=9.18$ by means of this independent method.

Since M 67 and Ruprecht 3 share similar distances and reddening values, the 2MASS photometric errors are comparable at a given magnitude and CMD sequences are expected to be similarly widened. The distribution of stars of Ruprecht 3 in Fig. 10 is a good match to that of M67. In particular, near the giant clump of M67 (Fig. 10), we see stars 1 and 3 (Fig. 1) of Ruprecht 3, which are known to be late-type stars (Sect. 4.2). Stars 5 and 6 (Fig. 1) were interpreted as turnoff stars $(\approx 1.5 \mathrm{Gyr})$ in Sect. 2. However, Fig. 10 suggests that they might be blue-stragglers of an older object such as M 67. Bluestragglers are brighter and bluer (hotter) than the MS turnoff of the majority of the cluster stars but lie along an apparent extension of the MS. At present, the leading explanation of the blue straggler phenomenon involves mass transfer in and/or merger of a binary system or the collision of stars, whether or not a binary is involved (Shetrone \& Sandquist 2000). According to this scenario, Ruprecht 3 could be considerably older, similar to M67 (3.5 Gyr) or even older like a globular cluster (Sect. 4.3). The MS of Ruprecht 3 (Fig. 10) appears to be severely depleted, which is a signature of a dynamically evolved object. 


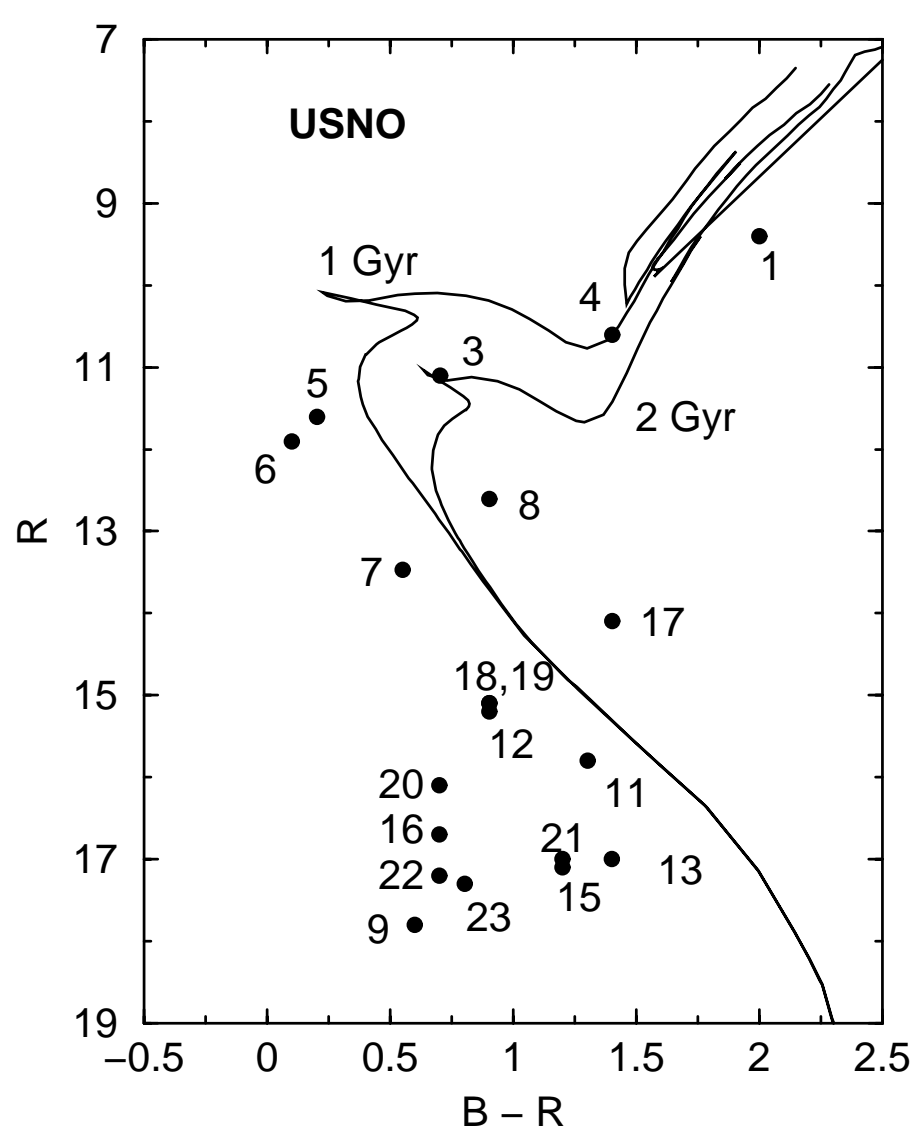

Fig. 4. $R \times(B-R)$ diagram for 20 stars in the Ruprecht 3 area from USNO photometry. Padova isochrones are shown.

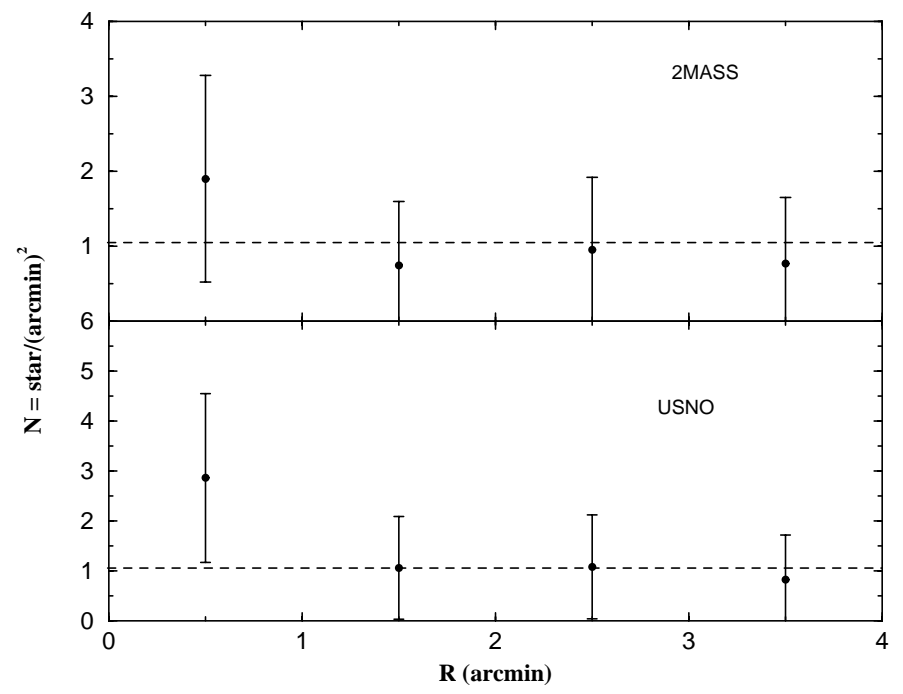

Fig. 5. 2MASS (upper panel) and USNO (lower panel) surface density in the area of Ruprecht 3 as a function of radius. Dashed lines are the mean of the surface density in offset fields.

\section{Concluding remarks}

We used 2MASS photometry in the $J, H$ bands complemented with the $B, R$ bands from USNO photographic photometry. The distributions of stars in both CMDs suggest physical evolutionary sequences, but contaminated by field stars. Thus, in the infrared photometry, we employed a statistical method to

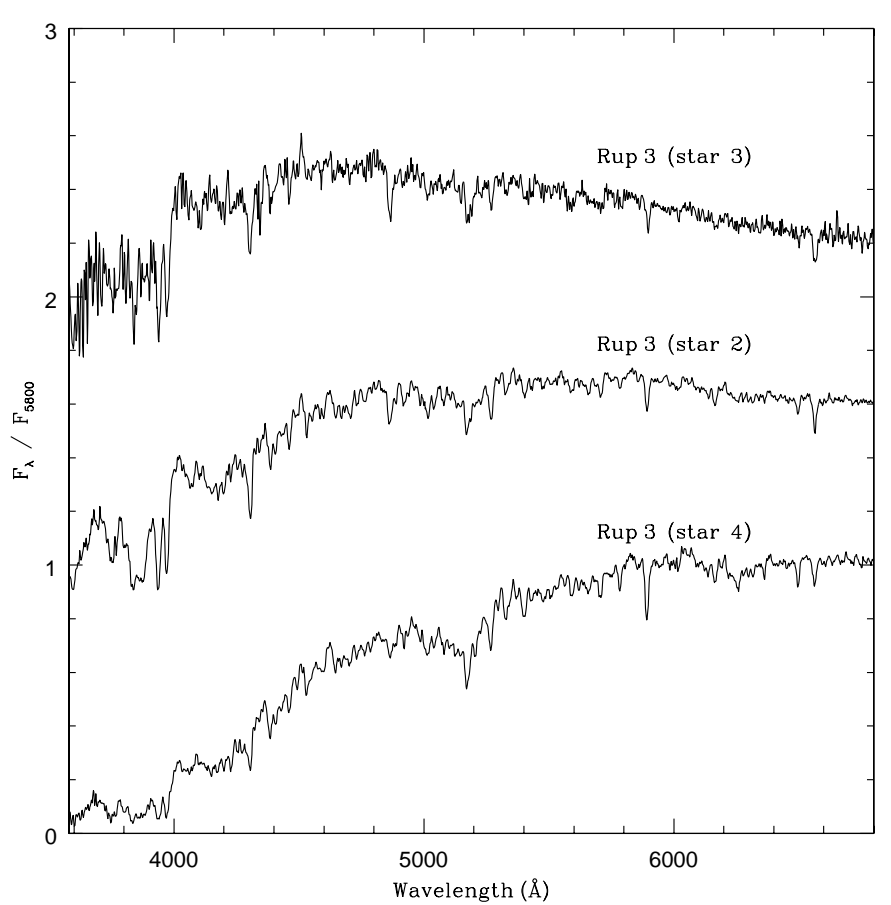

Fig. 6. The observed spectra of stars 2, 3 and 4 of Ruprecht 3. Spectra are in relative $F_{\lambda}$ units normalized at $5800 \AA$. Constants have been added to the spectra, except for the bottom one, for the sake of clarity.

remove this contamination. We analysed the structural properties of Ruprecht 3 by means of surface density profiles. The object is compact, with a maximum core radius of $1^{\prime}$, and it does not appear to have extensions, which is not unusual for a possible remnant.

The integrated spectrum appears to be remarkably similar to that of a relatively metal-rich globular cluster. However, it probably reflects a stochastic effect owing to the few brighter stars in the CMDs. Individual star spectroscopy was obtained for four bright stars and they turned out to be late-type, consistent with being giants from their loci in the CMDs.

From the various analyses, i.e. different CMDs, spectroscopy, comparison clusters and isochrones, the following conclusions may be drawn:

1. A reddening value $E(B-V)=0.04$ or $E(J-H)=0.013$ has been derived. The former is somewhat lower than the dust emission reddening value $E(B-V)_{F I R}=0.097 \mathrm{de}-$ rived by Schlegel et al. (1998) in the same direction. Since Ruprecht 3 is a relatively close object, the latter value also suggests the presence of some dust in the object's background.

2. An absolute distance modulus $(m-M)_{0}=9.28_{-0.10}^{+0.12}$, equivalent to a distance from the Sun $d_{\odot}=0.72_{-0.03}^{+0.04} \mathrm{kpc}$, has been derived. Assuming a solar Galactocentric distance of $8 \mathrm{kpc}$ (Reid 1993), we obtain: $X=-7.69 \mathrm{kpc}, Y=-0.59 \mathrm{kpc}$, $Z=-0.18 \mathrm{kpc}(X<0$ corresponds to our side of the Galaxy). Ruprecht 3 is then close to the Solar circle.

3. Interpreting the brighter blue stars as turnoff objects, we estimate an age of $1.5 \pm 0.5$ Gyr. Considering them to be blue-stragglers (consistent with more massive stellar products remaining in cluster remnants), Ruprecht 3 might be considerably older. 


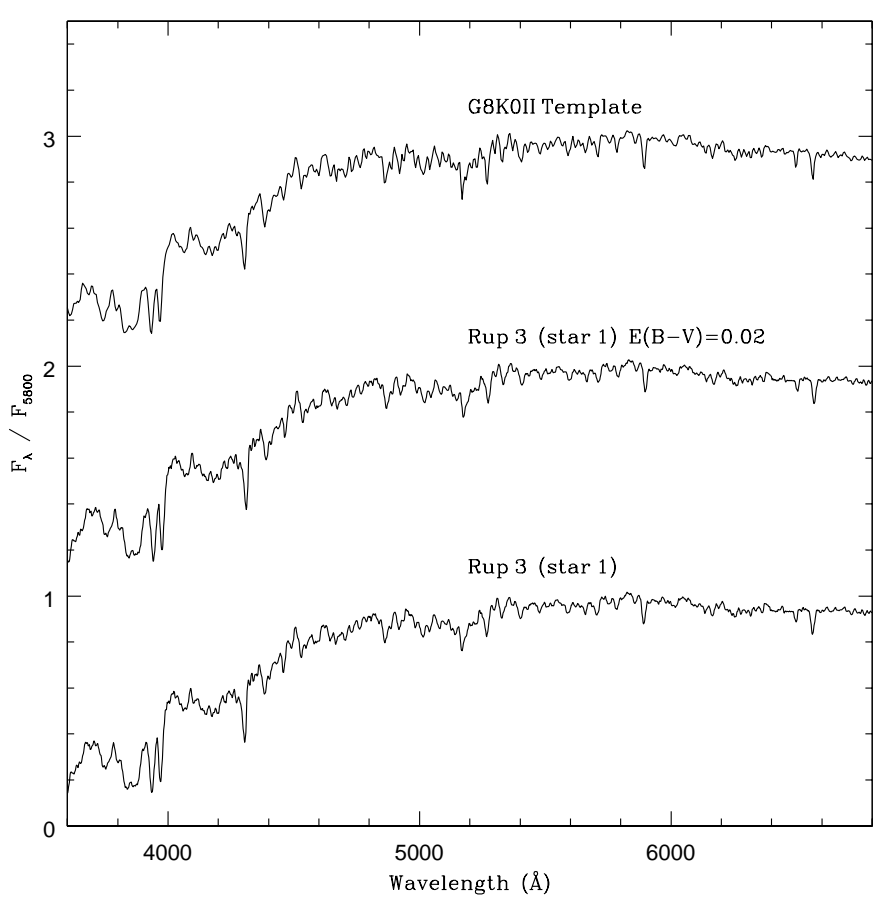

Fig. 7. Comparison of the observed (bottom) and reddening-corrected spectra of star 1 of Ruprecht 3 with the template (G8K0II) that best matches the spectrum. Constants have been added to the spectra for the sake of clarity, except for the bottom one. Units as in Fig. 6.

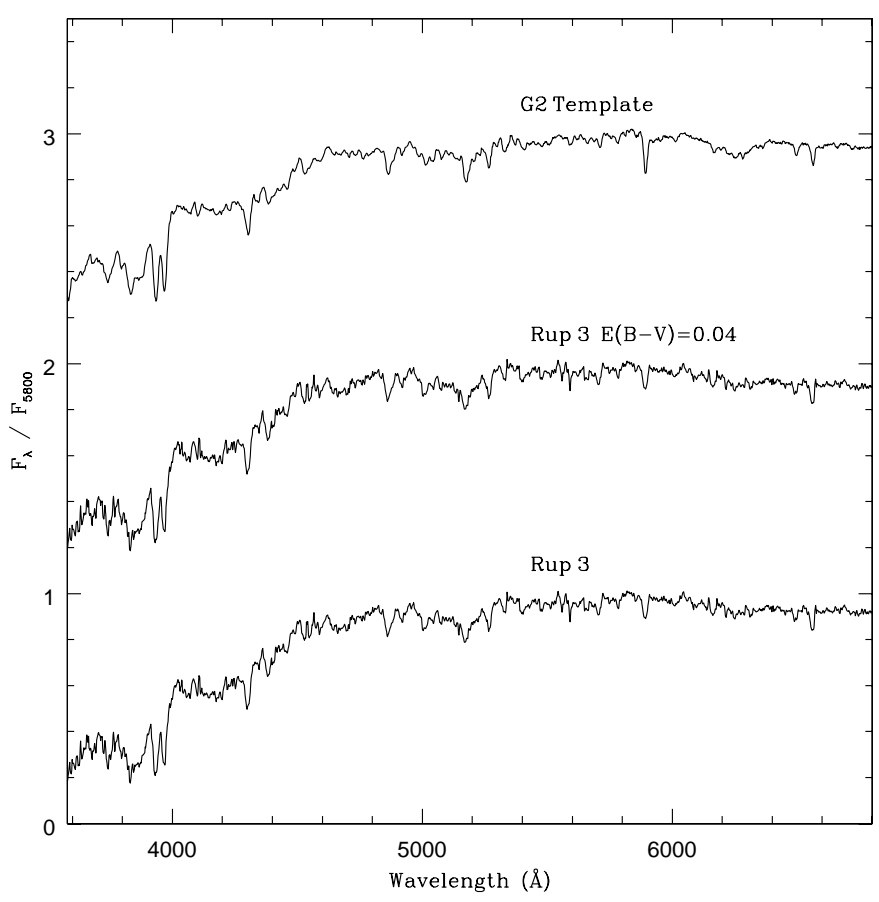

Fig. 8. The integrated spectrum of Ruprecht 3 , the same spectrum corrected for $E(B-V)=0.04$ and the globular cluster template $\mathrm{G} 2$. Additive constants were applied for the sake of clarity, except for the lower spectrum. Units as in Fig. 6.

The available photometries and spectroscopy of Ruprecht 3 suggest that it is probably an intermediate-age open cluster remnant with a compact structure containing 11 probable members and 5 less probable members.

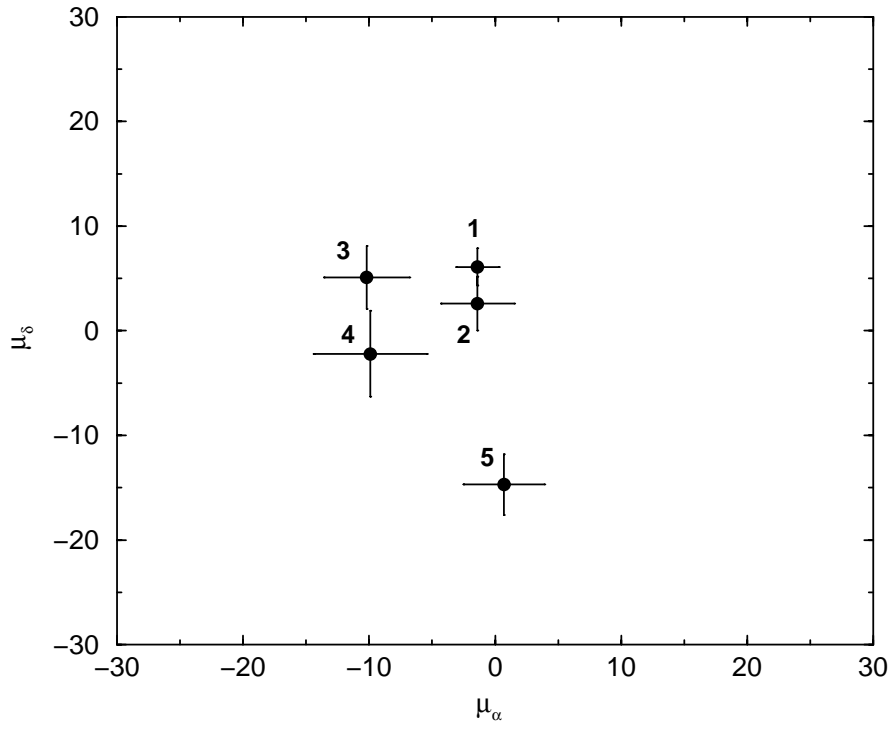

Fig. 9. Proper motions and respective error bars for five stars in the area of Ruprecht 3. Labels identify stars.

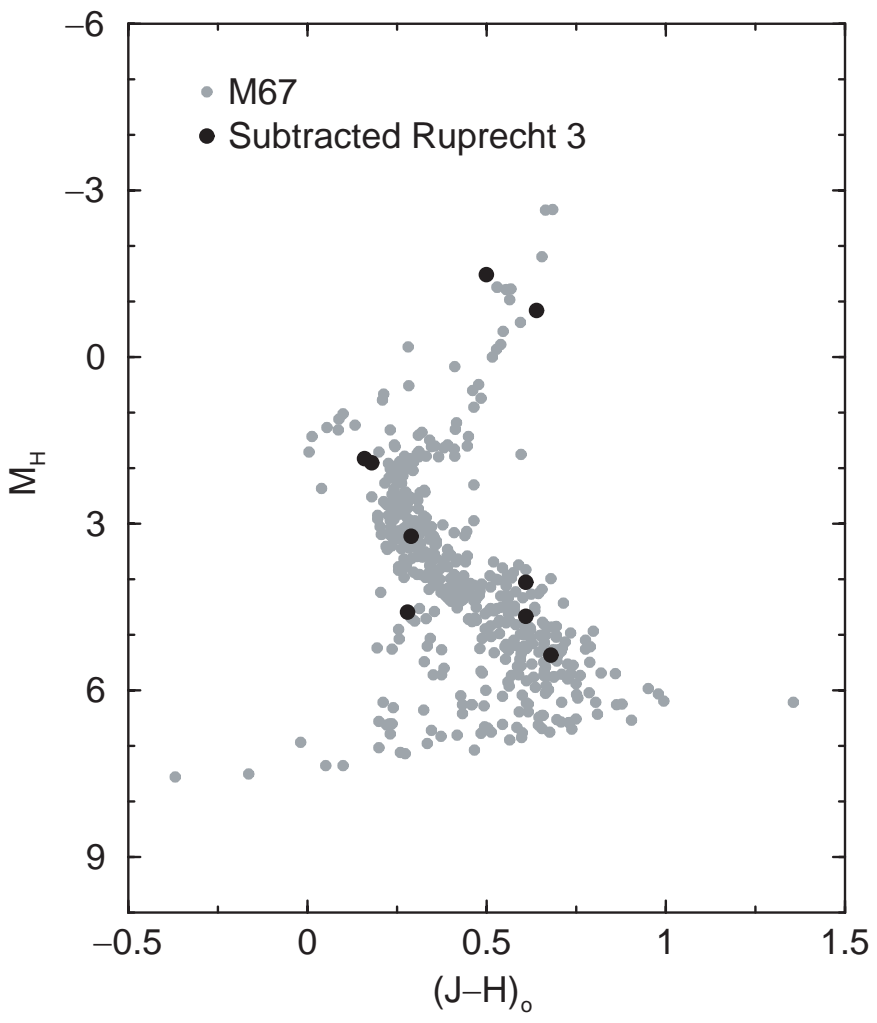

Fig. 10. Absolute infrared diagram for field subtracted Ruprecht 3 compared to that in the M 67 area from 2MASS photometry.

Acknowledgements. We thank L. O. Kerber and B. X. Santiago for the code of field statistical subtractions and for discussions. We are gratefully indebted to the CASLEO staff members and night assistants for their kind hospitality and support during the two observing runs. The authors acknowledge use of the $C C D$ and data adquisition system supported under U.S. National Science Foundation grant AST-90-15827 to R.M. Rich. Part of the present data was extracted from the CDS database (Strasbourg). This work was 
partially supported by the Brazilian institutios CNPq and CAPES and the Argentinian Institutions CONICET, Secyt (Universidad Nacional de Córdoba), Agencia Córdoba Ciencia and Agencia Nacional de Promoción Científica y Tecnológica (ANPCyT.) This publication makes use of data products from the Two Micron All Sky Survey, which is a joint project of the University of Massachusetts and the Infrared Processing and Analysis Center/California Institute of Technology, funded by the National Aeronautics and Space Administration and the National Science Foundation. The Guide Star Catalogue-II is a joint project of the Space Telescope Science Institute and the Osservatorio Astronomico di Torino.

\section{References}

Alter, G., Ruprecht, J., \& Vanysek, V. 1970, in Catalogue of Star Clusters and Associations + Supplements, 2nd ed. (Akademiai Kiado, Budapest)

Bassino, L. P., Waldhausen, S., \& Martinez, R. E. 2000, A\&A, 355, 138

Baumgardt, H. 1998, A\&A, 340, 402

Bica, E. 1988, A\&A, 195, 76

Bica, E., Santiago, B. X., Dutra, et al. 2001, A\&A, 366, 827

Cardelli, J. A., Clayton, G., \& Mathis, J. S. 1989, ApJ, 345, 245
Carraro, G. 2000, A\&A, 357, 145

Carraro, G. 2002, A\&A, 385, 471

de la Fuente Marcos, R. 1997, A\&A, 322, 764

de la Fuente Marcos, R. 1998, A\&A, 333, L27

Girardi, L., Bressan, A., Bertelli, G., \& Chiosi, C. 2000, A\&A, 141, 371

Kerber, L. O., Santiago, B. X., Castro, R., \& Valls-Gabaud, D. 2002, A\&A, 390, 121

Lauberts, A. 1982, in ESO/Uppsala Survey of the ESO (B) Atlas (ESO, Garching)

Minniti, D., Clariá, J. J., \& Gómez, M. 1989, Ap\&SS, 158, 9

Odenkirchen, M., \& Soubiran, C. 2002, A\&A, 383, 163

Olano, C. A., Poppel, W. G. L., \& Vota, M. D. 1980, IAUS, 85, 2470

Pavani, D. B. 2001, Estágios Finais da Evolução de Aglomerados Abertos, Msc Thesis, UFRGS, Porto Algre (Brasil)

Pavani, D. B., Bica, E., Dutra, C. M., et al. 2001, A\&A, 374, 554

Reid, M. N. A. 1993, ARA\&A, 31, 345

Ruprecht, J. 1966, Bull. Astron. Czechoslovakia, 17, 33

Schlegel, D. J., Finkbeiner, D. P., \& Davis, M. 1998, ApJ, 500, 525

Shetrone, M. D., \& Sandquist E. L. 2000, AJ, 120, 1913

Silva, D. R., \& Cornell, M. E. 1992, ApJS, 81, 865

Stone, R. P. S., \& Baldwin, J. A. 1983, MNRAS, 204, 347

Takahashi, K., \& Portegies Zwart, S. F. 2000, ApJ, 535, 759 